\title{
Spectrum management scheme for heterogeneous cognitive radio networks
}

\author{
Hassan Al-Mahdi'1,2, Yasser Fouad ${ }^{3}$ and Mohamed Kali1 ${ }^{3}$
}

\begin{abstract}
In cognitive radio networks (CRNs), opportunistic spectrum access (OSA) has been referred to as a solution to overcome the spectrum scarcity problem and to produce a better utilization of the existing licensed radio spectrum. The OSA spectrum management concept gives the secondary users (SUs) the opportunity to access unused parts of the licensed spectrum owned by primary users (PUs). In CRNs, the SUs are greatly suffering from the PUs activities. To reduce the impact of the PUs, we propose a spectrum management scheme over a non-homogenous spectrum environment (i.e. licensed and unlicensed) to improve the performance of the SUs. In the proposed scheme, different types of buffers are utilized to decrease the blocking probability for newly arriving users and dropping probability for ongoing connection as well as improving the throughput of SUs. An analytical model based a multi-dimensional continuous-time Markov chain and a level dependent quasi-birthdeath is introduced from which the aforementioned performance metrics are derived.
\end{abstract}

Keywords - Cognitive Radio Networks, Markov Chain, Spectrum Handoff.

\section{INTRODUCTION}

$\mathrm{C}$ ognitive radio networks (CRN) are intelligent networks which are capable to operate under a mixed spectrum environment that consists of both licensed and unlicensed bands [1]. Such kinds of networks are developed to overcome some issues that exist in both bands. In the licensed channels (LCs), there exist a large portion of the spectrum is temporally unused (i.e., white space). Thus, CRN can be deployed to exploit these spectrum white spaces efficiently by adding intelligence to each device to detect these white spaces and utilize them without interference with the primary networks (i.e. licensed network). In the unlicensed channels (UCs), there are varieties of important technologies such as IEEE 802.xx utilizing the industrial scientific and medical (ISM) band. With the growing proliferation of wireless devices, the spectrum efficiency of ISM band is decreasing. Thus, CRN can improve the efficiency in this band. The $\mathrm{CRN}$ are based on a spectrum management scheme called opportunistic spectrum access (OSA). OSA can be defined according to IEEE P1900.1 standard (IEEE Standard

[2], as follows: "Dynamic spectrum access by secondary users

1 is with Faculty of Sciences and Arts, Al Quryyat, Al Jouf University, Saudi Arabia, (e.mail: hassanwesf@yahoo.com)

2 is with Faculty of Computers and Information, Suez Canal University, Egypt.

3 is with the Computers and Information, Suez University, Egypt.
(SUs) that exploit local and instantaneous spectrum availability in a non-interfering manner and without primary user (PUs) negotiation". In the last few years, several analytical models are used to evaluate the performance of CRN based on OSA concept [3]-[6].

In [3], an analytical model based on a Markov chain analysis for spectrum access to LCs only has been presented. In addition, a simple channel reservation scheme for SUs has been proposed for reducing the dropping probability in the case of PU appearance. In [4] a spectrum sharing scheme for SUs coexisting with PUs has been evaluated through multidimensional Markov chain model in LCs. In [5], dynamic spectrum access schemes in the absence or the presence of buffering mechanism for the SUs have been proposed and analyzed. With buffering, they mean that instead of blocking the incoming SUs when the channels are busy, they have assumed that SUs inserted into a buffer until the channel(s) become idle. A Markov approach is developed to analyze the proposed spectrum sharing policies with generalized bandwidth size in both primary system and secondary system.

In [6], a spectrum sharing scheme with buffering for new SUs and interrupted SUs has been proposed. Markov model is used to describe and analyze the performance of the proposed scheme. In [7], steady-state analyses of spectrum handoff delay and channel availability have been presented when spectrum handoff is used in a cognitive radio network.

In [8], queue-based channel assembling strategies for multichannel CRNs as well as the analytical framework for performance evaluation of such networks are developed. Two queuing schemes, which consist of separate queues allocated for different traffic types with different priorities, have been developed. The developed analytical models are validated by extensive simulations.

In [9], a dynamic spectrum access scheme for CRNs, where priorities for the bandwidth, the spectrum access, and spectrum handoff are considered for three types of traffics is proposed. A multi-dimensional Markov chain with three state variables to analyze the state transitions of the scheme is developed.

The disadvantage of the all aforementioned schemes was that they evaluate the performance of CRN in the LCs only which do not agree with the definition of CRN. The CRN are networks which are networks operate under a mixed spectrum environment that consists of both LCs and UCs.

In [10], we have presented an analytical model based on 
Markov chain and a novel spectrum management scheme for CRN operated in both LCs and UCs. However, lots of details were hidden and presented in this work. In [11], the previous model presented in [10] is improved.

In this paper, the SU is operating in a heterogeneous environment of channels (i.e licensed and unlicensed) and utilizing different kinds of buffers to reduce the blocking probability for newly arriving users and dropping probability for ongoing connection. In addition, the classical users (CU) activities (i.e., the users which access UCs only) are taken into consideration. The system is modeled using a multidimensional continuous-time Markov chain and a level dependent quasi-birth-death, where the aforementioned performance metrics are obtained.

The rest of the paper is organized as follows: in section 2, the proposed scheme is presented. Section 3 describes the system occupancy of the proposed scheme. Furthermore, the performance metrics are introduced in section 4. Finally, the numerical results and conclusion are discussed in section 5 and 6.

\section{II.PROPOSED SCHEME DESCRIPTION}

In this section, the basic of the proposed spectrum management dynamics and common assumptions are introduced.

\section{A. Scheme Structure}

The proposed scheme consists of two stages:

- Stage 1 denotes the activities of both PUs and SUs on the LCs.

- $\quad$ Stage 2 denotes the activities of both SUs and CUs on the UCs.

Three types of users are competing on the available LCs and UCs. The first type called PUs which are wireless devices utilizing licensed frequency bands such as TV band. The second type called SUs which are wireless devices equipped with cognitive radio capabilities for adaptively and opportunistically accessing the available spectrum (i.e. LCs or UCs). The last type called CUs which are wireless devices without cognitive radio capabilities such as devices using the conventional standards e.g. IEEE 802.11 and Bluetooth. Figure 1 depicts the dynamics of SBSM scheme. As shown in the Figure, a newly arriving SU tries to access one of the idle LCs in the stage1. Upon its arrival, it may success to access an idle LC or get blocked. If it successes to access an idle LC in the stage 1, one of the following two cases can be happened:

- $\quad$ Case 1: the SU completes its transmission in stage 1 without any interruption.

- $\quad$ Case 2: the SU is dropped due to the appearance of a PU. The drooped SU tries to access an SU in stage 2.

If a newly arriving SU get blocked in stage 1 , it tries in the stage 2 . The CU, on the other hand, tries only in the stage 2 . It is noted that the PUs can be serviced only in the stage 1 . Figure 1 illustrates that stage 2 is highly affected by the activities of the PUs and SUs in stage 1. In the following, we formally state the model assumptions, which are largely reflected by the diagram in Figure 1.

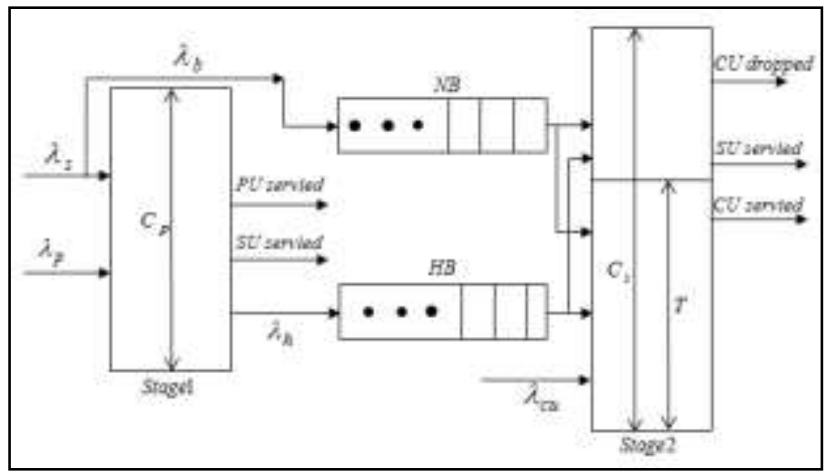

Fig. 1 The proposed spectrum management scheme

\section{B. Scheme Assumptions}

1. There are two types of available channels called LCs and UCs. The total number of channels $\mathrm{C}$ is given a $\mathrm{C}=C_{p}+C_{s}$, where $C_{p}$ and $C_{s}$ denote the number of available LCs and UCs respectively. Each SU is equipped with two finite buffers (waiting room) referred as $\mathrm{NB}$ and $\mathrm{HB}$ with size $N$ and $H$ respectively. The buffer $\mathrm{NB}$ is used to hold the blocked SUs from stage 1 and $\mathrm{HB}$ is used to hold dropped SUs from stage 1 if there are free spaces.

2. The LCs are shared between PUs and SUs, where a PU can preempt an ongoing SU if there is no idle LC.

3. The UCs are shared between SUs and CUs, where a SU can preempt an ongoing $\mathrm{CU}$ if there is no an idle $\mathrm{UC}$ and the number of ongoing CUs is less than a certain threshold $T$, where $T<C_{s}$.

4. Each PU, SU or CU requires only one channel for transmission.

5. A PU can be admitted if the LCs are not all occupied by other PUs (i.e., there is a vacant LC or there is at least one LC occupied by SU).

6. Since LCs are available in significant number, the newly arriving SUs use the following procedure as shown in Figure 1:

a. The SU access firstly one of LCs in stage 1 .

b. If all LCs are occupied, the SU is rejected with rate $\lambda_{b}$ and tries to utilize one of idle $\mathrm{UCs}$ (if any) in stage 2 given that $\mathrm{NB}$ is empty.

c. If there is no available UC and there is at least one UC occupied by $\mathrm{CU}$, the $\mathrm{CU}$ is dropped if the number of ongoing CUs is less than $T$. Otherwise, the newly arriving SU is pushed into the NB for later transmission utilizing one of UCs.

d. If there is no room in the NB then the newly arriving SU get blocked.

7. In case of PU appearance and there is no an idle LC, the SU should vacate its occupied LC and transfers into stage 2.The SU who vacates the channel for PU is called handoff SU. The handoff SU do one of the following: 
a. If the handoff buffer is empty then the SU performs a spectrum handoff to one of idle UCs. If there is no idle UCs and there is at least one UC occupied by $\mathrm{CU}$ given that the number of ongoing CUs is less than $T$ then $\mathrm{CU}$ is dropped and the $\mathrm{SU}$ is admitted. Otherwise, the handoff SUs will be queued in the HB for later transmission utilizing one of UCs.

b. If the HB is full, it will be dropped.

8. The arrivals process of PUs, SUs and CUs are assumed to be Poisson with rates $\lambda_{p}, \lambda_{s}$ and $\lambda_{c u}$ respectively. On the other hand, the service times of PU, SU and CU are assumed to be exponential with rates $\mu_{p}, \mu_{s}$ and $\mu_{c u}$ respectively.

The values of the rates $\lambda_{b}$ and $\lambda_{h}$ will be derived later in stage 1 analysis.

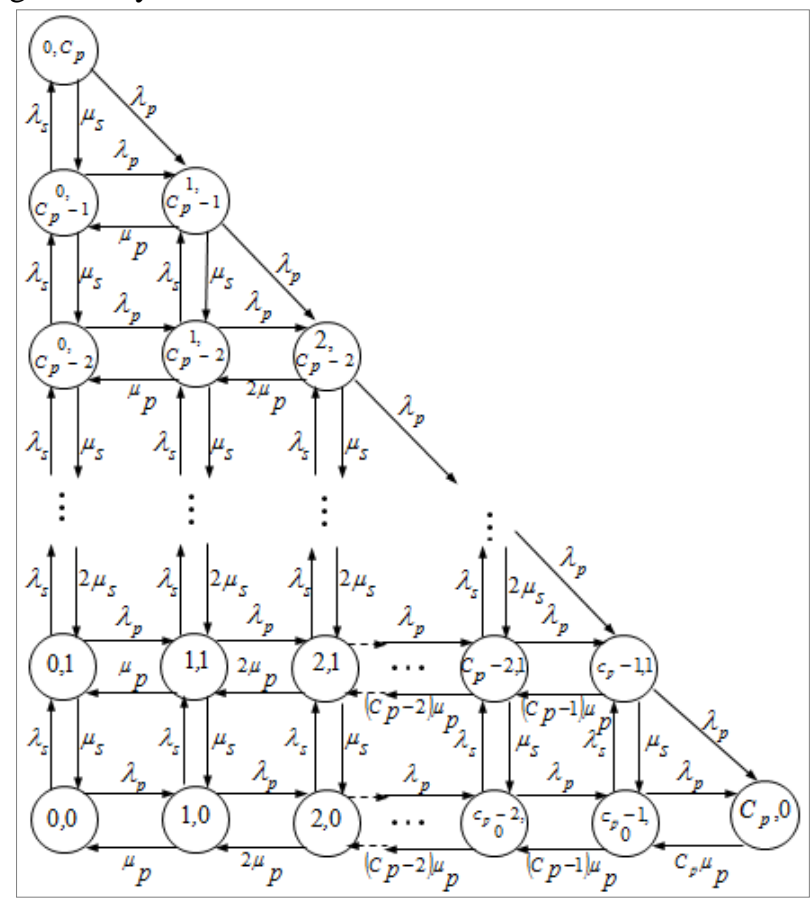

Fig. 2 The transition diagram of stage 1 .

\section{SYSTEM OCCUPANCY}

In this section, the system occupancy of the proposed scheme will be mathematically analyzed in both stage 1 and stage 2 . As aforementioned, stage 1 depicts the activities of both PUs and SUs on the LCs, while stage 2 depicts the activities of blocked SUs from stage 1, handoffs of SUs from stage 1 and CUs on the UCs.

\section{A. Stage 1}

The system in stage 1 can be modeled as a two-dimensional Markov chain with state space given as

$\mathrm{S}=\left\{(\mathrm{i}, \mathrm{j}), 0 \leq \mathrm{i} \leq \mathrm{C}_{\mathrm{p}}, \quad 0 \leq \mathrm{j} \leq \mathrm{C}_{\mathrm{p}}-\mathrm{i}\right\}$.

Where $i$ represents the number of active PU connections and $j$ represents the number of active SU connections. Figure 2 illustrates all possible transitions within the system. From this figure, the system can be considered as a level dependent quasi-birth-death (LQBD) [2] with $\mathrm{C}_{\mathrm{p}}+1$ levels, where the set of states in level $i$ is given as

$\mathrm{L}(\mathrm{i})=\left\{(\mathrm{i}, \mathrm{j}), \quad 0 \leq \mathrm{j} \leq \mathrm{C}_{\mathrm{p}}-\mathrm{i}\right\}$

In LQBD, transition from the state $(i, j)$ depend on the level $i$, where different levels have different numbers of states. The infinitesimal generator matrix $Q$ is sorted lexicographically and can then be grouped into finite sub-matrices as follows:

$\mathrm{Q}=\left[\begin{array}{cccccccc}\mathrm{A}_{1}^{0} & \mathrm{~A}_{0}^{0} & 0 & 0 & \cdots & 0 & 0 & 0 \\ \mathrm{~A}_{2}^{1} & \mathrm{~A}_{1}^{1} & \mathrm{~A}_{0}^{1} & 0 & \cdots & 0 & 0 & 0 \\ 0 & \mathrm{~A}_{2}^{2} & \mathrm{~A}_{1}^{2} & \mathrm{~A}_{0}^{2} & \cdots & 0 & 0 & 0 \\ \vdots & \vdots & \vdots & \vdots & \ddots & \vdots & \vdots & \vdots \\ 0 & 0 & 0 & 0 & \cdots & \mathrm{A}_{2}^{\mathrm{C}_{\mathrm{p}}-1} & \mathrm{~A}_{1}^{\mathrm{C}_{\mathrm{p}}-1} & \mathrm{~A}_{0}^{\mathrm{C}_{\mathrm{p}}-1} \\ 0 & 0 & 0 & 0 & \cdots & & \mathrm{A}_{2}^{\mathrm{C}_{\mathrm{p}}} & \mathrm{A}_{1}^{\mathrm{C}_{\mathrm{p}}}\end{array}\right]$

The sub-matrix $A_{0}^{i}$ denotes the transitions from level $i$ to leveli $+1, \mathrm{i}=0,1,2, \cdots, \mathrm{C}_{\mathrm{p}}-1$ with $\operatorname{dimension}\left(\mathrm{C}_{\mathrm{p}}+1-\right.$ $\mathrm{i}) \times\left(\mathrm{C}_{\mathrm{p}}-\mathrm{i}\right)$. The elements of the matrix $\mathrm{A}_{0}^{\mathrm{i}}$ are given as

$\mathrm{A}_{0}^{\mathrm{i}}=\left[\begin{array}{cccccc}\lambda_{\mathrm{p}} & 0 & 0 & 0 & \cdots & 0 \\ 0 & \lambda_{\mathrm{p}} & 0 & 0 & \cdots & 0 \\ 0 & 0 & \lambda_{\mathrm{p}} & 0 & \cdots & 0 \\ \vdots & \vdots & \vdots & \ddots & \vdots & \vdots \\ 0 & 0 & 0 & \cdots & \lambda_{\mathrm{p}} & \lambda_{\mathrm{p}} \\ 0 & 0 & 0 & \cdots & 0 & \lambda_{\mathrm{p}}\end{array}\right]$

The sub-matrix $A_{1}^{i}$ denotes the transitions within level $\mathrm{i}, \mathrm{i}=0,1,2, \cdots, \mathrm{C}_{\mathrm{p}} \quad$ with $\quad \operatorname{dimension}\left(\mathrm{C}_{\mathrm{p}}+1-\mathrm{i}\right) \times$ $\left(C_{p}+1-i\right)$. The elements of the sub-matrix $A_{1}^{i}$ are given as.

$\mathrm{A}_{1}^{\mathrm{i}}=\left[\begin{array}{cccccc}-\mathrm{q}_{0}^{\mathrm{i}} & \lambda_{\mathrm{s}} & 0 & \cdots & 0 & 0 \\ \mu_{\mathrm{s}} & -\mathrm{q}_{1}^{\mathrm{i}} & \lambda_{\mathrm{s}} & \cdots & 0 & 0 \\ 0 & 2 \mu_{\mathrm{s}} & -\mathrm{q}_{2}^{\mathrm{i}} & \cdots & 0 & 0 \\ \vdots & \vdots & \vdots & \ddots & \vdots & \vdots \\ 0 & 0 & 0 & \cdots & -\mathrm{q}_{\mathrm{C}_{\mathrm{p}}-1-\mathrm{i}}^{\mathrm{i}} & \lambda_{\mathrm{s}} \\ 0 & 0 & 0 & \cdots & \left(\mathrm{C}_{\mathrm{p}}-\mathrm{i}\right) \mu_{\mathrm{s}} & -\mathrm{q}_{\mathrm{C}_{\mathrm{p}}-\mathrm{i}}^{\mathrm{i}}\end{array}\right]$

where the values of elements $q_{j}^{i}$ are given as

$$
\mathrm{q}_{\mathrm{j}}^{\mathrm{i}}=\left\{\begin{array}{cl}
\lambda+\mathrm{i} \mu_{\mathrm{p}}+\mathrm{j} \mu_{\mathrm{s}} & \text { if } \quad 0 \leq \mathrm{i}<\mathrm{i}, \quad 0 \leq \mathrm{j} \leq \mathrm{C}_{\mathrm{p}}-\mathrm{i} \\
\lambda_{\mathrm{p}}+\mathrm{i} \mu_{\mathrm{p}}+\mathrm{j} \mu_{\mathrm{s}} & \text { if } \quad 0 \leq \mathrm{i}<\mathrm{i}, \mathrm{j}=\mathrm{C}_{\mathrm{p}}-\mathrm{i} \\
\mathrm{i} \mu_{\mathrm{p}} & \text { if } \quad \mathrm{i}=\mathrm{C}_{\mathrm{p}}, \mathrm{j}=0
\end{array}\right.
$$

Where $\lambda=\lambda_{p}+\lambda_{s}$ The sub-matrix $A_{2}^{i}$ denotes the transitions from level $i$ to leveli $-1, \mathrm{i}=1,2, \cdots, \mathrm{C}_{\mathrm{p}}$ with dimension $\left(C_{p}+1-i\right) \times\left(C_{p}+1-i\right)$. The elements of the matrix $A_{2}^{i}$ are given as

$\mathrm{A}_{2}^{\mathrm{i}}=\mathrm{i} \times\left[\begin{array}{cccccc}\mu_{\mathrm{p}} & 0 & 0 & 0 & \cdots & 0 \\ 0 & \mu_{\mathrm{p}} & 0 & 0 & \cdots & 0 \\ 0 & 0 & \mu_{\mathrm{p}} & 0 & \cdots & 0 \\ \vdots & \vdots & \vdots & \ddots & \vdots & \vdots \\ 0 & 0 & 0 & \cdots & \mu_{\mathrm{p}} & 0 \\ 0 & 0 & 0 & \cdots & 0 & \mu_{\mathrm{p}}\end{array}\right]$

The steady-state probabilities of stage 1 are organized lexicographically into the steady-state probability vector $\Pi$ as 
follows $\Pi=$

$\left[\pi_{0,0}, \pi_{0,1}, \cdots, \pi_{0, \mathrm{C}_{\mathrm{p}}}, \pi_{1,0}, \pi_{1,1}, \cdots, \pi_{1, \mathrm{C}_{\mathrm{p}}-1}, \cdots, \pi_{\mathrm{C}_{\mathrm{p}}, 0}\right] . \quad$ The vector $\Pi$ can be rewritten according to level $\mathrm{i}$ as follows $\Pi=$ $\left[\pi_{0}, \pi_{1}, \cdots, \pi_{C_{p}}\right], \quad$ where $\pi_{i}=\left[\pi_{0,0}, \pi_{0,1}, \cdots, \pi_{0, C_{p}-i}\right] \cdot$ The steady state distributions $\pi_{0}, \pi_{1}, \cdots, \pi_{\mathrm{C}_{\mathrm{p}}}$ can be obtained by solving the system equations

$\Pi \mathrm{Q}=0$

and the normalization condition

$\Pi Q=\sum_{i=0}^{C_{p}} \pi_{i} 1=0$.

Where $1=[1,1, \cdots, 1]^{\mathrm{t}}$ is a unit vector with appropriate size. Using equations (3)-(7), the system equations in (8) and (9) can be solved by applying the algorithmic approaches [13], where the sub-matrices in Q change from level to level. Once the probabilities $\pi_{i, j}$ are obtained for $0 \leq \mathrm{i} \leq \mathrm{C}_{\mathrm{p}}$ and $0 \leq \mathrm{i} \leq$ $\mathrm{C}_{\mathrm{p}}, \quad 0 \leq \mathrm{j} \leq \mathrm{C}_{\mathrm{p}}-\mathrm{i}$, the rates $\lambda_{\mathrm{b}}$ and $\lambda_{\mathrm{h}}$ are obtained as follows,

$\lambda_{\mathrm{b}}=\sum_{\mathrm{i}=0}^{\mathrm{C}_{\mathrm{p}}} \pi_{\mathrm{i}, \mathrm{C}_{\mathrm{p}}-\mathrm{i}}$

and

$\lambda_{\mathrm{h}}=\frac{\sum_{\mathrm{i}=0}^{\mathrm{C}_{\mathrm{p}}-1} \pi_{\mathrm{i}, \mathrm{C}_{\mathrm{p}}-\mathrm{i}}}{\lambda_{\mathrm{s}}\left(1-\lambda_{\mathrm{b}}\right)}$

\section{B. Stage 2}

Due to the bi-class nature of stage 2, we identify four variables of system occupancy k, l, m, and $n$ whichrefer to the number of ongoing SUs, the number of ongoing CUs which occupy UCs, the number of SUs in HB and the number of SUs stored in the NB respectively. The process of spectrum access to UCs is modeled as a 4-dimensional Markov chain $\mathrm{k}, \mathrm{l}, \mathrm{m}, \mathrm{n}$ with state space $\mathrm{S}$ given as:

$$
\begin{gathered}
\mathrm{S} 2=\left\{(\mathrm{k}, \mathrm{l}, \mathrm{m}, \mathrm{n}), 0 \leq \mathrm{k} \leq \mathrm{C}_{\mathrm{s}}, \quad 0 \leq \mathrm{l} \leq \mathrm{C}_{\mathrm{s}}-\mathrm{k}, \quad 0\right. \\
\leq \mathrm{m} \leq \mathrm{H}, \quad 0 \leq \mathrm{n} \leq \mathrm{N}\}
\end{gathered}
$$

The transitions between all possible states are triggered by SUs and CUs arrivals, SUs and CUs departures as well as queued handoff SU and new SU events. The system dynamics under the aforementioned assumptions can be written as

$\mathcal{M}_{\mathrm{k}, \mathrm{l}, \mathrm{m}, \mathrm{n}} \mathrm{p}_{\mathrm{k}, \mathrm{l}, \mathrm{m}, \mathrm{n}}=\mathcal{F}_{\mathrm{k}, \mathrm{l}, \mathrm{m}, \mathrm{n}}$.

Where $\mathcal{M}_{\mathrm{k}, \mathrm{l}, \mathrm{m}, \mathrm{n}}$ and $\mathcal{F}_{\mathrm{k}, \mathrm{l}, \mathrm{m}, \mathrm{n}}$ describe the flux out and flux in state $(\mathrm{k}, \mathrm{l}, \mathrm{m}, \mathrm{n})$ respectively and $\mathrm{p}_{\mathrm{k}, \mathrm{l}, \mathrm{m}, \mathrm{n}}$ denoting the probability that the system in state $(\mathrm{k}, \mathrm{l}, \mathrm{m}, \mathrm{n})$, where $\mathrm{p}_{\mathrm{k}, \mathrm{l}, \mathrm{m}, \mathrm{n}}=$ Ofor $\mathrm{k}<0, \mathrm{l}<0, \mathrm{~m}<0, \mathrm{n}<0$ or $\mathrm{k}>\mathrm{C}_{\mathrm{s}}, \mathrm{l}>\mathrm{C}_{\mathrm{s}}, \mathrm{m}>$ $\mathrm{H}, \mathrm{n}>\mathrm{N}$. The value of $\mathcal{M}_{\mathrm{k}, \mathrm{l}, \mathrm{m}, \mathrm{n}}$ can be written as follows,

$$
\begin{gathered}
\mathcal{M}_{\mathrm{k}, \mathrm{l}, \mathrm{m}, \mathrm{n}}=\lambda_{\mathrm{b}} \delta_{\mathrm{k}, \mathrm{l}, \mathrm{m}, \mathrm{n}}^{1}+\lambda_{\mathrm{h}} \delta_{\mathrm{k}, \mathrm{l}, \mathrm{m}, \mathrm{n}}^{2}+\lambda_{\mathrm{cu}} \delta_{\mathrm{k}, \mathrm{l}, \mathrm{m}, \mathrm{n}}^{3}+\mathrm{k} \mu_{\mathrm{s}} \\
+\mathrm{l} \mu_{\mathrm{cu}}
\end{gathered}
$$

Where $\lambda=\lambda_{\mathrm{b}}+\lambda_{\mathrm{h}}$ and $\delta_{\mathrm{k}, \mathrm{l}, \mathrm{m}, \mathrm{n}}^{\mathrm{i}}$ is defined as $\delta_{\mathrm{k}, \mathrm{l}, \mathrm{m}, \mathrm{n}}^{1}= \begin{cases}1 \quad \text { if } \quad \begin{array}{l}\mathrm{k}+\mathrm{l}<\mathrm{C}_{\mathrm{s}}, \mathrm{m}=0, \mathrm{n}=0 \\ \\ \\ \end{array} \quad \begin{array}{l}\mathrm{Vk}+\mathrm{l}=\mathrm{C}_{\mathrm{s}}, \mathrm{m}=0, \mathrm{n}=0, \mathrm{l} \geq \mathrm{T} \\ \mathrm{Vk}+\mathrm{l}=\mathrm{C}_{\mathrm{s}}, \quad 0 \leq \mathrm{n}<\mathrm{N}, \mathrm{l}<\mathrm{T} \\ \text { otherwise }\end{array}\end{cases}$

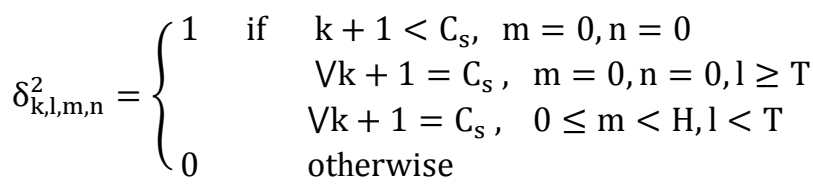

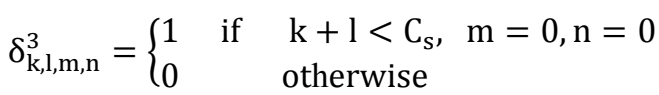

The value of $\mathcal{F}_{\mathrm{k}, \mathrm{l}, \mathrm{m}, \mathrm{n}}$ can be written as follows,

$$
\begin{aligned}
\mathcal{F}_{\mathrm{k}, \mathrm{l}, \mathrm{m}, \mathrm{n}}=\left(\lambda_{\mathrm{b}}+\right. & \left.\lambda_{\mathrm{h}}\right)\left(\chi_{\mathrm{k}-1, \mathrm{l}, \mathrm{m}, \mathrm{n}}^{1} \mathrm{p}_{\mathrm{k}-1, \mathrm{l}, \mathrm{m}, \mathrm{n}}\right. \\
& \left.+\chi_{\mathrm{k}-1, \mathrm{l}+1, \mathrm{~m}, \mathrm{n}}^{2} \mathrm{p}_{\mathrm{k}-1, \mathrm{l}+1, \mathrm{~m}, \mathrm{n}}\right) \\
& +\lambda_{\mathrm{h}} \chi_{\mathrm{k}, \mathrm{l}, \mathrm{m}-1, \mathrm{n}}^{3} \mathrm{p}_{\mathrm{k}, \mathrm{l}, \mathrm{m}-1, \mathrm{n}} \\
& +\lambda_{\mathrm{b}} \chi_{\mathrm{k}, \mathrm{l}, \mathrm{m}, \mathrm{n}-1}^{4} \mathrm{p}_{\mathrm{k}-1, \mathrm{l}+1, \mathrm{~m}, \mathrm{n}} \\
& +\lambda_{\mathrm{cu}} \chi_{\mathrm{k}, \mathrm{l}-1, \mathrm{~m}, \mathrm{n}}^{5} \mathrm{p}_{\mathrm{k}, \mathrm{l}-1, \mathrm{~m}, \mathrm{n}} \\
& +(\mathrm{k}+1) \mu_{\mathrm{s}} \mathrm{p}_{\mathrm{k}+1, \mathrm{l}, \mathrm{m}, \mathrm{n}} \\
& +(\mathrm{l}+1) \mu_{\mathrm{cu}} \mathrm{p}_{\mathrm{k}, \mathrm{l}+1, \mathrm{~m}, \mathrm{n}}
\end{aligned}
$$

where

$$
\begin{aligned}
& \chi_{\mathrm{k}, \mathrm{l}, \mathrm{m}, \mathrm{n}}^{1}=\left\{\begin{array}{lll}
1 & \text { if } & \mathrm{k}+1<\mathrm{C}_{\mathrm{s}}, \mathrm{m}=0, \mathrm{n}=0, \\
0 & \text { otherwise }
\end{array}\right. \\
& \chi_{\mathrm{k}, \mathrm{l}, \mathrm{m}, \mathrm{n}}^{2}=\left\{\begin{array}{lll}
1 & \text { if } & \mathrm{k}+1=\mathrm{C}_{\mathrm{s}}, \mathrm{m}=0, \mathrm{n}=0, \mathrm{l}>\mathrm{T} \\
0 & & \text { otherwise }
\end{array}\right.
\end{aligned}
$$

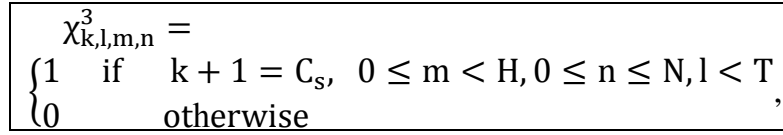

$$
\begin{aligned}
& \chi_{\mathrm{k}, \mathrm{l}, \mathrm{m}, \mathrm{n}}^{4}= \\
& \left\{\begin{array}{ll}
1 & \text { if } \\
0 & \mathrm{k}+1=\mathrm{C}_{\mathrm{s}}, \quad 0 \leq \mathrm{m} \leq \mathrm{H}, 0 \leq \mathrm{n}<\mathrm{N}, \mathrm{l}<\mathrm{T}
\end{array},\right. \\
& \text { and } \\
& \chi_{\mathrm{k}, \mathrm{l}, \mathrm{m}, \mathrm{n}}^{5}=\left\{\begin{array}{lll}
1 & \text { if } & \mathrm{k}+1<\mathrm{C}_{\mathrm{s}}, \quad \mathrm{m}=0, \mathrm{n}=0 \\
0 & & \text { otherwise }
\end{array}\right.
\end{aligned}
$$

By substituting from equations (14) and (15) in (13), the balance equation of stage 2 system dynamics is obtained. Since it is a complicated to drive a closed form for $\mathrm{p}_{\mathrm{k}, \mathrm{l}, \mathrm{m}, \mathrm{n}}$, we apply the iterative algorithm introduced in (M. A. Kalil and et al, 2013) to get the stationary distribution of probabilities $\mathrm{p}_{\mathrm{k}, \mathrm{l}, \mathrm{m}, \mathrm{n}}$.

\section{PERFORMANCE METRICS}

\section{A. SU Blocking Probability}

As we mentioned before, The PUs have higher priority with pre-emption power to access the LCs over the SUs. Hence a SU get blocked in stage 1 if all LCs are occupied. On the other hand, the blocked SU from stage 1 can be blocked again in stage 2 if all UCs are occupied and the NB is full. In such case 
the blocking probability of SUs con be written as,

$B_{S U}=\lambda_{b} \sum_{j=0}^{C_{s}} \sum_{m}^{H} \psi_{C_{s}-j} p_{j, C_{s}-j, m, N}$

Where the values of indicator variable $\psi_{\mathrm{C}_{\mathrm{s}}-\mathrm{j}}$ is defined as

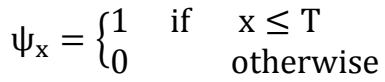

\section{B. Dropping Probability}

Upon the arrival of a PU, it searches for an idle LC. If there is no an idle LC, it will preempt an ongoing SU (if any). The preempted SU transfers to the stage 2. If there is no an idle UC and the $\mathrm{HB}$ is full then the dropped SU from stage 1 will be lost at stage 2 . In such case the dropping probability of a SU is given as follows,

$\operatorname{Drop}_{S U}=\lambda_{h} \sum_{j=0}^{C_{s}} \sum_{n}^{N} \psi_{C_{s}-j} p_{j, C_{s}-j, H, n}$

\section{The SU Throughput}

The throughput can be defined as the product of the number of successful SU connections per unit time in stage 1 and stage 2 and the service time per completed connection. Thus, the throughput $W_{1}$ and $W_{2}$ of stage 1 and stage 2 can be written respectively as follows,

$\mathrm{W}_{1}=\frac{\lambda_{\mathrm{s}}\left(1-\lambda_{\mathrm{b}}\right)\left(1-\lambda_{\mathrm{h}}\right)}{\mathrm{C}_{\mathrm{p}} \mu_{\mathrm{s}}}$

and

$\mathrm{W}_{2}=\frac{\lambda_{\mathrm{s}}\left[\left(1-\mathrm{B}_{\mathrm{SU}}\right)+\left(1-\text { Drop }_{\mathrm{SU}}\right)\right]}{\mathrm{C}_{\mathrm{s}} \mu_{\mathrm{s}}}$

Hence, the total the throughput $\mathrm{W}$ can be written from (17) and (18) as $\mathrm{W}=\mathrm{W}_{1}+\mathrm{W}_{2}$.

\section{V.NUMERICAL RESULTS}

In this section, the numerical results are presented to evaluate the performance of the proposed scheme. The following operational parameters are used: $\lambda_{\mathrm{s}}=0.2, \mu_{\mathrm{s}}=$ $0.3, \mu_{\mathrm{p}}=0.3$ and $\mu_{\mathrm{cu}}=0.2$. The values of $\mathrm{H}, \mathrm{N}$ and $\lambda_{\mathrm{P}}$ are varied. Moreover, different CUs arrival rate $\lambda_{\mathrm{cu}}$ are introduced to show the effects of the CUs in both low and high traffic. For a fair comparison, we evaluate the performance of the proposed SBSM scheme when $\mathrm{C}_{\mathrm{p}}=8$ and $\mathrm{C}_{\mathrm{s}}=0$ (i.e. stage 1 only) and when $C_{p}=4$ and $C_{s}=4$ (i.e. stage 1 and stage 2) to show the effects when the SUs utilize the LCs of stage 1 only and when they utilize both stage 1 and 2 .

Figure 3 illustrates the SU blocking probability versus the PU traffic load. Moreover, different buffer sizes are generated to measure the effects of buffer size on the SUs' performance. On the other hand, the Figure measures the blocking probability when the equipped buffers are removed from the SUs (i.e. $\mathrm{NB}=0$ and $\mathrm{HB}=0$ ). From the Figure, we note that the blocking probability is improved with the proposed SBSM scheme.

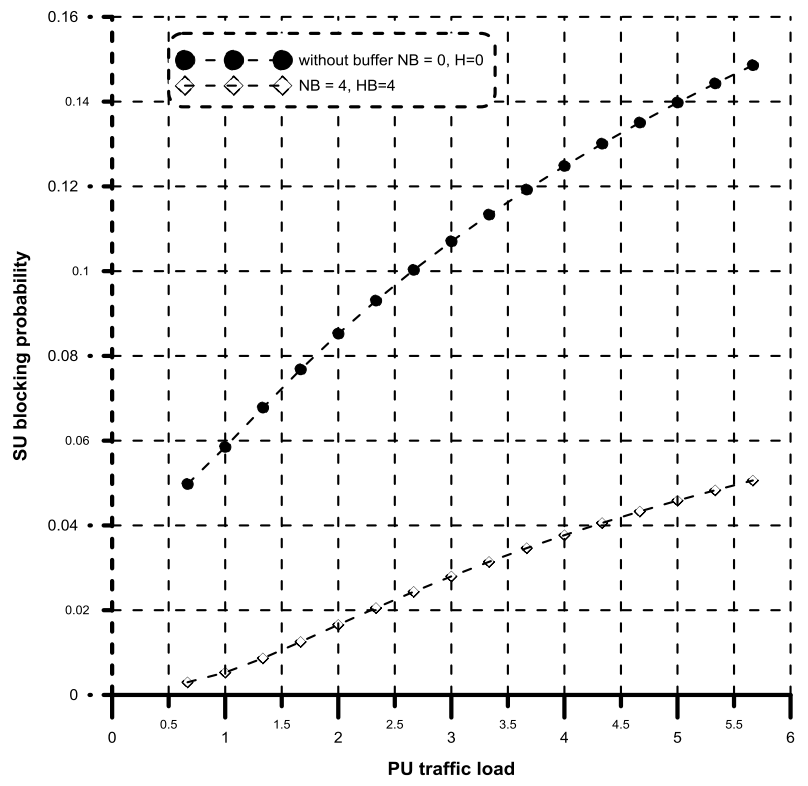

Fig 3 SU blocking probability with and without buffering.

Figure 4 illustrates the effects of the CU on the blocking probability of SU in both low and high traffic of CUs. Although a CU has a negative effects on the blocking probability of SU, the proposed SBSM scheme is still out performs those schemes that are operate in homogenous environment (i.e. $\mathrm{C}_{\mathrm{s}}=0$ and no buffering).

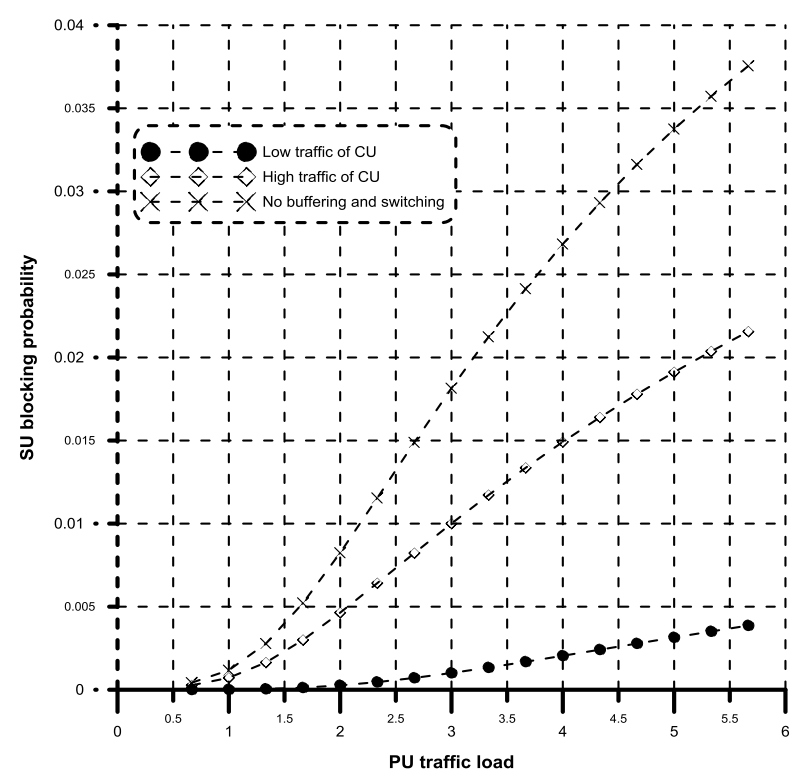

Fig 4. The effects of the CUs on the SU blocking probability.

Figure 5 illustrates the dropping probability of SUs as a function of the traffic load of the PUs. From Figure we note that, increasing the traffic load of PUs increases the dropping probability of a SU and the dropping probability of a SU is improved with the presence of buffers in stage 2 . If $\mathrm{N}=0$ and $\mathrm{H}=0$ in stage 2 then more SUs that are blocked from stage 1 will be blocked in stage 2. Similarly, more SUs that are 
dropped from stage 1 will be blocked in stage 2 also.

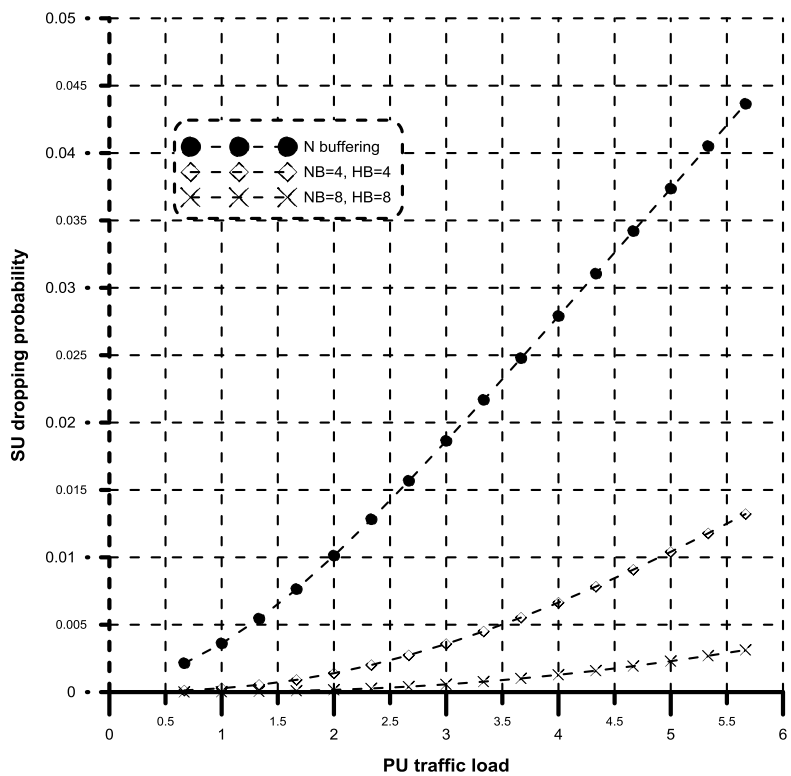

Fig. 5 The SU dropping probability with and without buffering.

Figure 6 plots the dropping probability of SUs as a function of the traffic load of PU. From this figure we note that, the dropping probability increases when our proposed scheme is reduced to stage 1 which introduced in literatures

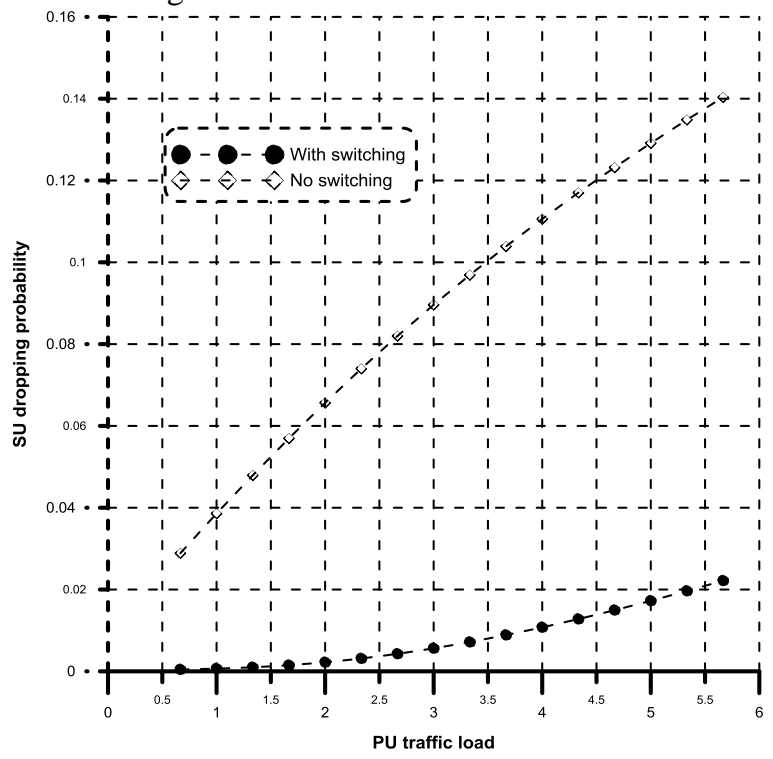

Fig. 6 The SU dropping probability with and without switching.

Finally, Figure 7 illustrates the SU throughput of the proposed scheme as a function of traffic load of PU. From the Figure we note that, the SU throughput decreases when eliminated the switching and buffering control (i.e. SUs use stage 1 only).

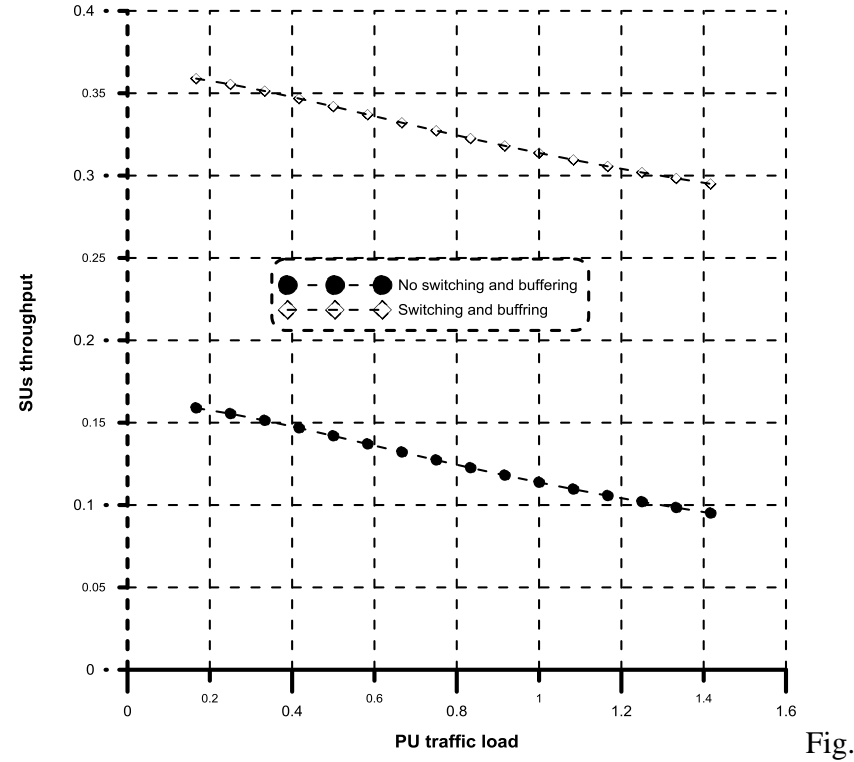

7 SUs' throughput as a function of traffic intensity for PUs.

\section{REFERENCES}

[1] I. F. Akyildiz, W.-Y. Lee, M. C. Vuran, and S. Mohanty, (2006). Next generation/ dynamic spectrum access/cognitive radio wireless networks: A survey, Computer Networks Journal (Elsevier), vol. 50, no. 13, pp. 2127_2159.

[2] IEEE Standard Deffinitions and Concepts for Dynamic Spectrum Access: Terminology Relating to Emerging Wireless Networks, System Functionality, and Spectrum Management, IEEE Std 1900.1-2008 Std.

[3] X. Zhu, L. Shen, and T.-S. Yum, (2007). Analysis of cognitive radio spectrum access with optimal channel reservation," IEEE Commun. Lett., vol. 11, no. 4, pp. 304-306. https://doi.org/10.1109/LCOM.2007.348282

[4] P. K. Tang, Y. H. Chew, L. C. Ong, and M. K. Haldar, (2006) Performance of secondary radios in spectrum sharing with prioritized primary access, in Proc. of Military Communications Conference (MILCOM 2006), Washington, D.C, USA, pp. 1_7. https://doi.org/10.1109/milcom.2006.302214

[5] Y. Xing, R. Chandramouli, S. Mangold, and S. S. N, (2006). Dynamic spectrum access in open spectrum wireless networks,_ IEEE Journal on Selected Areas in Communications, vol. 24, no. 3, pp. 626-637. https://doi.org/10.1109/JSAC.2005.862415

[6] C. P. T. Hong, Y. Lee, , and I. Koo, (2010). Spectrum sharing with buffering in cognitive radio networks, in Proc. of the 2nd International Conference Intelligent Information and Database Systems (ACIIDS 2010), Hue City, Vietnam, pp. 261-270.

[7] D. Lee, and W. Yeo, (2015). Channel availability analysis of spectrum handoff in cognitive radio networks.IEEE Commun. Lett., vol. 19, no. 3, pp. 435-438. https://doi.org/10.1109/LCOMM.2014.2387415

[8] Indika A. M. Balapuwaduge, Lei Jiao, Vicent Pla, and Frank Y. Li, (2014). Channel Assembling with Priority-Based Queues in Cognitive Radio Networks: Strategies and Performance Evaluation. IEEE Trrans, On Wireless Communications, vol. 18, no. 7, pp 630-644 https://doi.org/10.1109/TWC.2013.120713.121948

[9] Thi My Chinh Chu, Hoc Phan, and Hans-Jürgen Zepernick (2014). Dynamic Spectrum Access for Cognitive Radio NetworksWith PrioritizedTraffics. IEEE Commun. Lett., vol. 11, no. 4, pp. 1218-1221.

[10] H. Al-Mahdi, M. A. Kalil, F. Liers and A. Mitschele-Thiel, (2009). Increasing spectrum capacity for ad hoc networks using cognitive radios: an analytical model," IEEE Commun. Lett., vol 13, no. 9, pp. 676-678. https://doi.org/10.1109/lcomm.2009.090103

[11] M. A. Kalil, H. Al-Mahdi and A. Mitschele-Thiel. (2013). Performance evaluationof secondary users operating on a heterogeneous spectrum environment,Wireless Pers. Commun., vol.72, 2251-2262 https://doi.org/10.1007/s11277-013-1147-3. 
[12] Bolch, G., Greiner, S., de Meer, H. and Trivedi, K.S., (2006). Queueing networks and Markov chains: modeling and performance evaluation with computer science applications. John Wiley \& Sons. https://doi.org/10.1002/0471791571

[13] Latouche, Guy, and Vaidyanathan Ramaswami. (1999). Introduction to matrix analytic methods in stochastic modeling. Vol. 5. Siam, 1999. https://doi.org/10.1137/1.9780898719734 